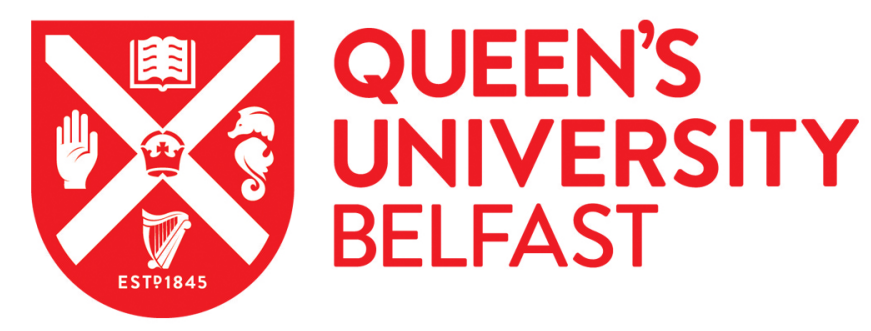

\title{
Load case characterization for the aircraft structural design process
}

Dharmasaroja, A., Armstrong, C. G., Murphy, A., Robinson, T. T., McGuinness, S. H. M., lorga, N. L., \& Barron, J. R. (2017). Load case characterization for the aircraft structural design process. AIAA Journal, 55(8), 27832792. https://doi.org/10.2514/1.J055544

\section{Published in:}

AIAA Journal

\section{Document Version:}

Peer reviewed version

Queen's University Belfast - Research Portal:

Link to publication record in Queen's University Belfast Research Portal

\section{Publisher rights}

Copyright $\odot 2017$ by the American Institute of Aeronautics and Astronautics, Inc. This work is made available online in accordance with the publisher's policies. Please refer to any applicable terms of use of the publisher.

\section{General rights}

Copyright for the publications made accessible via the Queen's University Belfast Research Portal is retained by the author(s) and / or other copyright owners and it is a condition of accessing these publications that users recognise and abide by the legal requirements associated with these rights.

Take down policy

The Research Portal is Queen's institutional repository that provides access to Queen's research output. Every effort has been made to ensure that content in the Research Portal does not infringe any person's rights, or applicable UK laws. If you discover content in the Research Portal that you believe breaches copyright or violates any law, please contact openaccess@qub.ac.uk. 


\title{
Load case characterization for the aircraft structural design process
}

\author{
A. Dharmasaroja ${ }^{1}$, C.G. Armstrong ${ }^{2}$, A. Murphy ${ }^{3}$, T.T. Robinson ${ }^{4}$ \\ Queen's University Belfast, Belfast, UK, BT95AG \\ S.H.M. McGuinness ${ }^{5}$, N.L. Iorga ${ }^{6}$ \\ Airbus, Bristol, UK, BS34 7AT \\ and \\ J.R. Barron ${ }^{7}$ \\ Airbus, Toulouse, France, 31060
}

\begin{abstract}
Since aircraft undergo countless ground and flight scenarios during their service life, a large volume of global load cases must be analyzed in a Global Finite Element Model (GFEM) to identify the structural load paths and internal load distributions. Analyzing all load cases is not possible due to computational constraints, so that substantial engineering judgement in load case down-selection is required. An approach is proposed to reduce the number of load cases analyzed by using Singular Value Decomposition (SVD) to derive a smaller set of characteristic loads which approximates all the global load distributions. Herein robust error quantification methods are also proposed to allow the definition of the
\end{abstract}

\footnotetext{
${ }^{1}$ School of Mechanical and Aerospace Engineering, Queen's University, Belfast, UK. adharmasaroja01@qub.ac.uk

2 c.armstrong@qub.ac.uk

3 a.murphy@qub.ac.uk

${ }^{4}$ t.robinson@qub.ac.uk

${ }^{5}$ shaun.mcguinness@airbus.com

${ }^{6}$ nicolae.iorga@airbus.com

${ }^{7}$ Airbus France, 31060 Toulouse, France. james.barron@airbus.com
} 
characteristic loads to a targeted accuracy level, so that the calculation of structural failure can also be evaluated with a known precision. The major downstream benefit of the process is that the analysis results for this set of characteristic loads can be superimposed to create the internal load distributions resulting from all the original load cases, with known accuracy. For realistic design and load data, internal loads need to only be calculated for a few tens of characteristic loads rather than thousands of real load cases.

\section{Nomenclature}

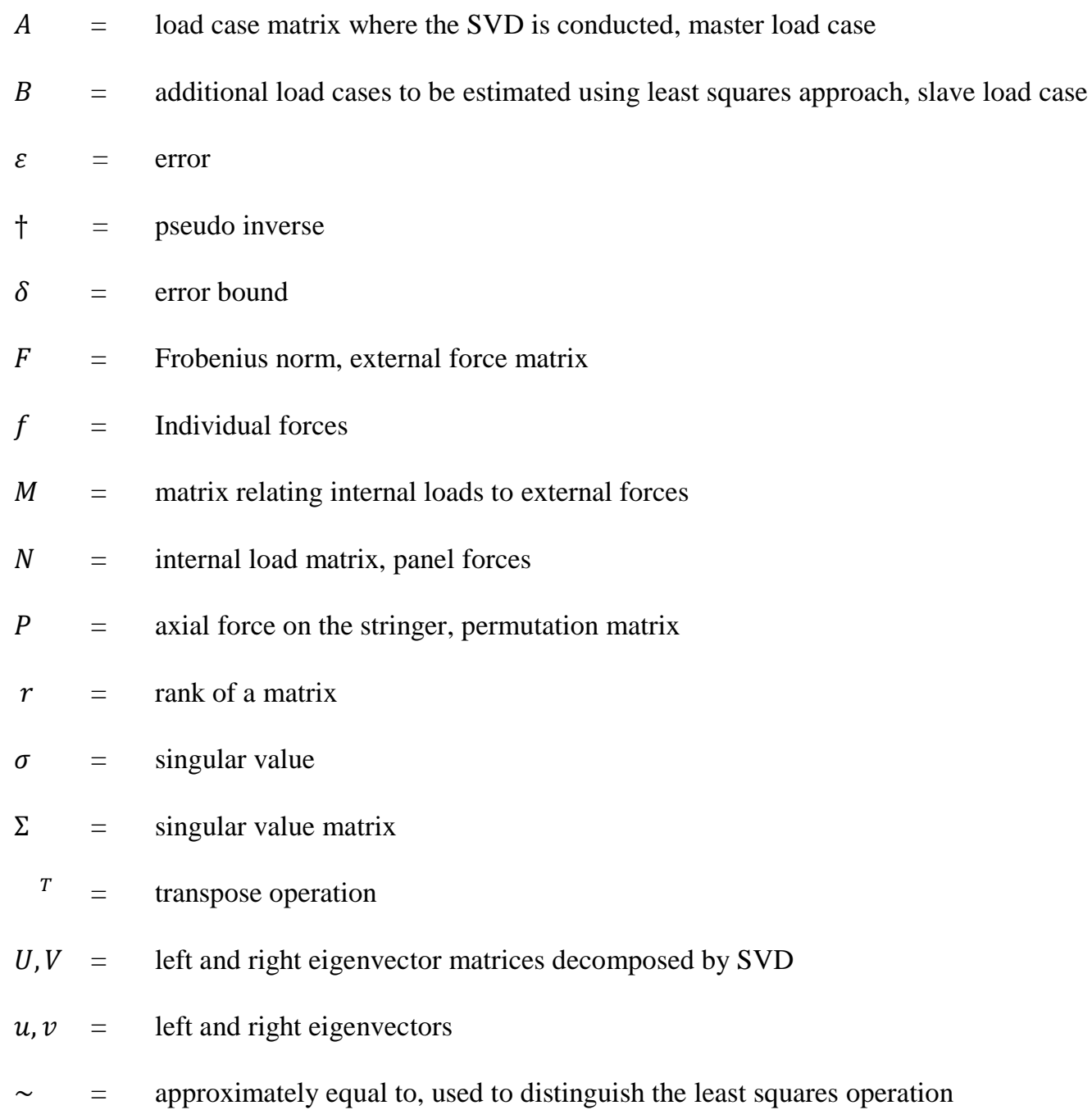




\section{Introduction}

uring the aerospace design process external aircraft loads must be predicted and analyzed to ensure the safe and efficient operation of an aircraft. Generally, airworthiness authorities require certain specific cases to be considered. It is known from experience however that these 'book cases' do not cover the full expanse of the design and operating envelope. Therefore, many additional load cases must be analyzed [1], [2]. These cases are typically summarized for subsequent structural sizing activity in an integrated form e.g. 6 degree of freedom (DOF) force and moment components at reference load stations along the wing and fuselage. Note that many other interesting quantities (IQs) may also be calculated such as local acceleration, air pressures etc., but for this work only the 6 DOF force values are of interest.

What results from the examination of all the book, design and operating envelope cases is the response of the aircraft to dynamic maneuvers with potentially millions of load cases, each representing a pseudo-static external loading on the aircraft for a given time step during a maneuver. The number of 6DOF force and moment load cases is sometimes beyond $10^{6}[3]$. The vast number of cases developed cannot be analyzed in the subsequent structural sizing activity in which each analysis requires significant effort. In addition, it is highly probable that the examined load cases include both duplicate states of load and states of load which are insignificant with regards to the structural design.

As it is not possible to analyze in detail all load cases, some form of down-selection must be implemented in order to identify a reduced set of critical load cases. The most notable methods are load envelopes which are typically based on maximum and minimum criteria [2], [4] and [5]. These include 1-dimensional criteria such as maximum or minimum individual force and moment components, local accelerations or local lift coefficients etc. Additional 2-dimensional down-selection criteria may also be implemented. These would include the convex hull of the shear vs. torque envelope at each wing station (to capture critical cases for spar shear flow) and moment vs. torque (to indicate the maximum state of loading in the covers). These 2-dimensional criteria are typically implemented at the wing root and mid-span regions and are related to the design, e.g. an additional station may be considered at the wing-tip if a wing-tip device is employed. A clear disadvantage from all these methods is that the down-selection process does not take into account any structural analysis directly.

Another method is to consider the stress or strain response of carefully chosen local elements. Again, the method does not actually reflect the failure of the structure but the influence of the internal loads. Additionally, down- 
selection is only performed on selected structural elements based on engineering judgment. A number of studies have been identified in the field of load case down-selection. For example a study conducted by Turkish Aerospace [6] highlights the need to incorporate calculations of reserve factor (the applied load divided by the allowable load on a structure) within the critical load selection process. Robust, efficient and automatic load case down-selection, ideally based on structural strength calculations, is an open problem in aircraft design.

As part of an investigation focusing on load case down-selection for structural sizing and optimization within the aerospace industry, a novel robust mathematical method of characterizing a large set of load cases has been developed [7]. The developed approach makes use of Singular Value Decomposition (SVD) [8], a matrix decomposition method commonly used in data processing and pattern recognition.

In Section II an overview of SVD and its adaption to structural loading is presented before the examples demonstrating aircraft wing problems are given. In Section III the Global Finite Element Model (GFEM) analysis using the characterized loads is demonstrated, and it is shown that these external loads generate an even smaller number of internal load patterns on a given structural feature. The results are discussed and the paper concludes with a summary of the benefits and potential extensions of the method.

\section{Characterization of Wing Loading by Singular Value Decomposition}

The usefulness of matrix decompositions, which factorise a matrix into a product of other matrices, is particularly highlighted when a large array of data is concerned. They can often identify hidden patterns inside a matrix and give useful insight into its structure. One of the most well-known approaches is SVD. In this section the background theory of the SVD will be explained followed by its application to aircraft load case down-selection.

\section{A. Singular Value Decomposition}

SVD decomposes a general rectangular matrix into more fundamental forms. Supposing $A$ is a real $\mathrm{m}$ by $\mathrm{n}$ matrix, the SVD of $A$ can be expressed as

$\begin{array}{rrrcl}A & = & U & \Sigma & V^{T} \\ \underset{(\mathrm{m} \times \mathrm{n})}{ } & & \underset{(\mathrm{m} \times \mathrm{m})}{(\mathrm{m} \times \mathrm{n})} & \underset{(\mathrm{n} \times \mathrm{n})}{ }\end{array}$

such that $\Sigma$ is a diagonal matrix containing singular values $\sigma$ arranged in order of magnitude. $U$ and $V$ are left and right eigenvector matrices which consist of a set of $u$ and $v$ orthonormal vectors (unit and orthogonal). These 
orthonormal vectors are in $\mathbb{R}^{m}$ (column space) and $\mathbb{R}^{n}$ (row space) of the matrix respectively. Equation (1) can alternatively be expressed in terms of vectors as

$$
A=\left[\begin{array}{lllllll}
u_{1} & \cdots & u_{r} & \mid & u_{r+1} & \cdots & u_{m}
\end{array}\right]\left[\begin{array}{ccc|c}
\sigma_{1} & & & \\
& \ddots & & 0 \\
& & \sigma_{r} & \\
\hline & 0 & & 0
\end{array}\right]\left[\begin{array}{c}
v_{1}^{T} \\
\vdots \\
v_{r}^{T} \\
\hline v_{r+1}^{T} \\
\vdots \\
v_{n}^{T}
\end{array}\right]
$$

These vectors are all linearly independent. Therefore, the SVD identifies the orthonormal bases which are the smallest set of orthonormal vectors required to describe the row and column spaces of the matrix and prioritizes their magnitudes by the singular value matrix. For a matrix $A$ of rank $r$, there are $r$ non-zero singular values. In the present context, this means the vectors which correspond to the singular values beyond $r$ i.e. $u_{r+1} \cdots u_{m}$ and $v_{r+1} \cdots v_{n}$ do not contribute any new load case information and so are redundant. The data in $A$ is fully preserved when the $\mathrm{r}$ singular values are linearly combined with the $\mathrm{u}$ and $\mathrm{v}$ vectors.

\section{B. Reduced rank approximation}

As previously shown in Eq. (1), the matrix $A$ can be constructed from the compact set of singular values and eigenvectors with $r$ components, where $r$ designates the full rank of the matrix. The number $r$ is normally equal to the smallest dimension of the $A$ matrix (either rows or columns). In fact the singular values $\sigma_{i}$ as $i$ approaches $r$ could be negligibly small. Supposing the SVD of $A$ to be

$$
A=\left[\begin{array}{lllllll}
u_{1} & \cdots & u_{k} & \mid & u_{k+1} & \cdots & u_{r}
\end{array}\right]\left[\begin{array}{ccc|ccc}
\sigma_{1} & & & & & \\
& \ddots & & & 0 & \\
& \sigma_{k} & & \\
\hline & & & \sigma_{k+1} & & \\
& 0 & & & \ddots & \\
& & & & & \sigma_{r}
\end{array}\right]\left[\begin{array}{c}
v_{1}^{T} \\
\vdots \\
v_{k}^{T} \\
\hline v_{k+1}^{T} \\
\vdots \\
v_{r}^{T}
\end{array}\right]
$$

If $\sigma_{k} \gg \sigma_{k+1}$, the singular values from $\sigma_{k+1}$ onwards will have a very small effect therefore the $A$ matrix can be approximated from

$$
A \approx A_{k}=U_{k} \Sigma_{k} V_{k}^{T}
$$

such that $A_{k}$ is the approximation of the original matrix $A$ produced from a reduced dimension of SVD products: $U_{k} \Sigma_{k} V_{k}^{T}$. This is often called the reduced rank approximation and was introduced by Eckart and Young [9] as a means to reduce the redundancy in a matrix. 


\section{Error Quantification}

When the reduced rank approximation is applied to the SVD, there is always an approximation error. In order to implement this technique, it is necessary to be able to mathematically predict how many singular values are required in the approximation in order to achieve the target threshold error.

The error arising from the reduced rank approximation can be quantified using Frobenius norm $\left(\|\cdot\|_{F}\right)$, which is the square-root of the sum of the squares of all elements in the matrix. From the fact that $U$ and $V^{T}$ are invariant under the Frobenius norm [10], the Frobenius norm of a matrix $A$ is equal to the Frobenius norm of its singular value matrix decomposed from the SVD by

$$
\|A\|_{F}=\left\|U \Sigma V^{T}\right\|_{F}=\|\Sigma\|_{F}=\sqrt{\sigma_{1}^{2}+\cdots+\sigma_{r}^{2}}
$$

This equation indicates that the Frobenius norm of $A$ depends only on all singular values determined by the SVD. The Frobenius norm of the approximation error can be determined from the ignored singular values by

$$
\left\|A-A_{k}\right\|_{F}=\left\|\Sigma_{r-k}\right\|_{F}=\sqrt{\sigma_{k+1}^{2}+\cdots+\sigma_{r}^{2}}
$$

such that the $r-k$ represents the partitioning of the $\Sigma$ matrix from $k^{\text {th }}$ rank to $r^{\text {th }}$ rank.

As a result, the Frobenius norm estimator of the error, expressed as a percentage between the reduced rank approximation and the original matrix can be derived as

$$
\frac{\left\|A-A_{k}\right\|_{F}}{\|A\|_{F}}=\frac{\left\|\Sigma_{r-k}\right\|_{F}}{\|\Sigma\|_{F}}=\sqrt{\frac{\sigma_{k+1}^{2}+\cdots+\sigma_{r}^{2}}{\sigma_{1}^{2}+\cdots+\sigma_{r}^{2}}}
$$

\section{Characteristic loads}

In the current aircraft load and structural design process, load cases are normally provided by the loads department in terms of an array of data. For example in wing design, the loads are commonly represented as 6 DOF of forces and moments at each of " $j$ " stations along the wing span. Thus an array of " $i$ " load cases will contain “ $i$ " rows and " $6 \times j$ " columns. Typically these 6 DOFs are three forces and three moments in the $x, y, z$ axes at each single loading station. Denote the force at the $1^{\text {st }} \mathrm{DOF}(x$ axis) as

$$
F_{x}=\left[\begin{array}{cccc}
f_{11} & f_{12} & \cdots & f_{1 j} \\
f_{12} & f_{22} & \cdots & f_{2 j} \\
\vdots & \vdots & \ddots & \vdots \\
f_{i 1} & f_{i 2} & \cdots & f_{i j}
\end{array}\right]
$$


such that $i$ is the number of load cases, and $j$ is the number of load stations on the finite element model. Therefore a 6DOF load case array can be expressed by

$$
A=\left[\begin{array}{l|l|l|l}
F_{x} & F_{y} & \cdots & M_{z}
\end{array}\right]
$$

The number of rows in $A$ is exactly the same as the number of load cases in Eq. (8). The number of columns is equal to the number of loaded stations multiplied by 6 DOFs. The number of rows which represents the number of load cases can be varied whereas the number of columns which represents the number of load stations is constant.

As each of the rows (load cases) is effectively a vector of force and moment values, a load case matrix can be formed with columns representing the components of force and moment at each wing station and each row being a load case or load distribution. It is possible that this set of load cases contains duplicate or redundant load cases which could be represented as linear combinations of other cases, thus unnecessarily increasing the number of load cases and the subsequent design and analysis effort. The reduced rank SVD can be employed to detect and remove these redundancies in the matrix, provided that it is arranged appropriately. In other words, the number of rows can be reduced using the SVD.

Referring to Eq. (1), the SVD of $A$ produces three matrices: $U, \Sigma$, and $V^{T}$. Each of the orthonormal basis vectors in $U$ and $V^{T}$ is scaled by the corresponding singular value $(\sigma)$. By combining $\Sigma$ and $V^{T}$, the data will be organized in a way that the most important bases of the row space (load case space) are classified as

$$
L=\Sigma V^{T}
$$

or in terms of vectors as

$$
\left[\begin{array}{c}
l_{1} \\
\vdots \\
l_{r}
\end{array}\right]=\left[\begin{array}{c}
\sigma_{1} v_{1}^{T} \\
\vdots \\
\sigma_{r} v_{r}^{T}
\end{array}\right]
$$

such that $L$ is the matrix of characteristic loads containing characteristic load vectors $l_{1} \cdots l_{r}$. The characteristic load vectors prioritize the basis vectors in the load space $\left(v^{T}\right)$ by the corresponding singular values $(\sigma)$.

Since the last few singular values may be negligible, the reduced rank approximation expressed in Eq. (4) can be applied to Eq. (10) as

$$
L_{k}=\Sigma_{k} V_{k}^{T}
$$

Substituting Eq. (12) into (4), the load case matrix $(A)$ can be approximated from the characteristic loads as

$$
A \approx U_{k} L_{k}
$$


Thus, the full set of load cases $A$ can be approximated by linear combination of a much smaller set of characteristic loads $L_{k}$. This has significant benefits in terms of the analysis of the wing and its structural features, which will be outlined in Section III.

\section{E. Adding new load cases into the characteristic loads}

As a design evolves, there are often additional load cases to be analyzed. It is useful to find the representation of these new load cases as a linear combination of the existing characteristic loads, and to determine how good the approximation is. This section will show how any new load cases can be estimated from the existing characteristic loads.

It has already been shown that the matrix of characteristic loads extracted from the original loading matrix can be used to approximate the original loads. The new situation arises when there is a new load case matrix, which contains the additional load cases to be analysed in the new design cycle.

Supposing characteristic loads $(L)$ extracted from the original load case matrix $(A)$ already represent all the load patterns in a structure, it should be possible to represent a set of new load cases denoted as $(B)$ by

$$
B=\widetilde{U} L
$$

such that $\widetilde{U}$ is the left eigenvector matrix that corresponds with the $L$ extracted from the original loading to create the new matrix. The addition of the $\sim$ sign above $\widetilde{U}$ does not signify any mathematical operation but distinguishes the matrix from the normal $U$ product that could also be obtained directly from the SVD of $B$ itself. Given that $B$ is provided, and $L$ has already been obtained from the previous design cycle, the only unknown $\widetilde{U}$ must be determined so as to linearly relate $L$ and $B$ together.

Transposing Eq. (14) gives

$$
L^{T} \widetilde{U}^{T}=B^{T}
$$

Equation (15) can be solved for $\widetilde{U}^{T}$ if the inverse of $L^{T}$ exists. However, $L^{T}$ is often rectangular, and hence cannot be inverted. Instead, the pseudo inverse ${L^{T}}^{\dagger}$, which is the generalisation of the inverse matrix [11], can be used to solve for $\widetilde{U}^{T}$ of the linear system. Using the transposition properties of the pseudo inverse $\left(L^{T^{\dagger}}=L^{\dagger^{T}}\right)$, Eq. (15) can be expressed as

$$
\widetilde{U}^{T} \approx L^{\dagger^{T}} B^{T}
$$

The left eigenvector matrix of the new load case matrix can be computed by transposing Eq. (16) as 


$$
\widetilde{U} \approx B L^{\dagger}
$$

This equation implies that the new left eigenvector matrix $(\widetilde{U})$ can be computed using the pseudo inverse of the existing characteristic load matrix $\left(L^{\dagger}\right)$. Therefore, the new load case matrix $(B)$ can be approximated based on the linear combination between $\widetilde{U}$ and the existing characteristic loads $(L)$.

Substituting Eq. (17) into Eq. (14) and applying the reduced rank approximation, the new matrix $B$ can be approximated from $k$ existing characteristic load cases as

$$
B_{k} \approx \widetilde{U}_{k} L_{k}=B L_{k}^{\dagger} L_{k}
$$

\section{F. Method demonstrator on wing external load cases - description}

The aircraft loading data being used in this experiment was generated from the loads department in Airbus (UK) with appropriate desensitising (i.e. scaling and modification to preserve commercial confidentiality). There are initially a total of 274 load cases for 27 wing stations where the 6-DOF loads are located at the centre of each rib bay, resulting in $274 \times 162$ values. The load cases were generated from a typical real aircraft database containing both ground and flight load cases, and were similar to one that would commonly be used in the actual design process. As the labels for each load case were removed for proprietary reasons the load cases have not been correlated with any physical phenomena.

The data was arbitrarily separated into two sets: a master set and a slave set, in order to imitate the scenario where the loading is updated in a next design cycle. The 100 load cases in the master set are assumed to be loadings in the first cycle, and the 174 load cases in the slave set are assumed to be loadings from the next cycle. Both sets of load cases were then re-arranged into the desired format. Denoting the master set as $A$ and slave set as $B$, the size of each set is defined as follows:

$$
\begin{aligned}
& A=(100 \times(27 \times 6))=(100 \times 162) \\
& B=(174 \times(27 \times 6))=(174 \times 162)
\end{aligned}
$$

The experiment is divided into three parts. Firstly the results when the SVD was directly performed on the master set are shown. The relevant error quantification methods were applied to investigate the error occurring from the approximation. Secondly the experiment when all 274 load cases were included in $A$ is also included. Thirdly the SVD was directly performed on the slave set. The pseudo inverse was applied to transform the loadings in the slave set into the existing characteristic load spaces. 
The SVD was computed using the predefined SVD function in MATLAB, which is computationally efficient. To illustrate, the time used to compute the SVD of a matrix with one million entries is less than 0.1 milliseconds on a standard PC with a Quad-core processor.

\section{G. Method demonstrator on wing external load cases - master load cases \& all load cases}

The SVD products decomposed from the original matrix $(A)$ were used to calculate the reduced rank characteristic loads $L_{k}$ as described by Eq. (12). The Frobenius norm percentage error estimator of the errors due to approximating $A$ from $k$ characteristic loads were subsequently determined by using Eq. (7) and plotted as a function of $k$ as shown in Fig. 1.

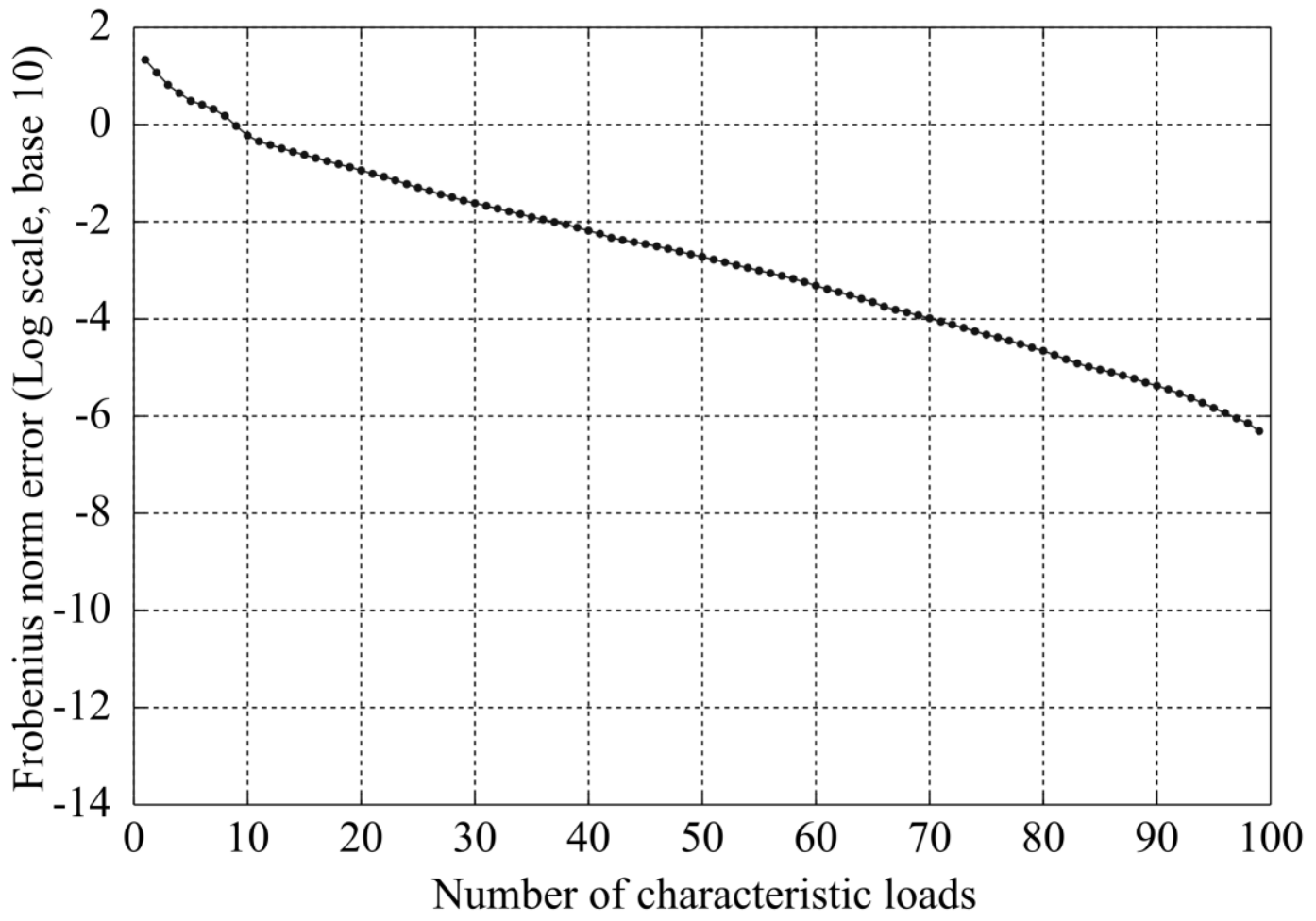

Fig. 1 Frobenius norm \% error, master set (log scale). When $k=100$ the original matrix is recovered.

Examining Fig. 1, the approximation error computed from the first characteristic load (L) alone (i.e. k=1) produces the maximum error at around $22 \%$ and the error reduces as the number of characteristic loads increases. For 25 characteristic loads the relative error between the reduced rank approximation and the original matrix, as calculated by the Frobenius norm, is equal to approximately $0.1 \%$. As the Frobenius norm only estimates the approximation error of the entire matrix, the accuracy of individual forces and moments was investigated. The 
reduced rank approximation preserves the singular values with high magnitudes, so the load cases with high value magnitudes should be approximated most accurately. The two load cases with the smallest and highest magnitudes in terms of Euclidean norm ${ }^{1}$ were selected as examples as they were expected to represent the worst and the best fitting results. The comparison plot between the actual and approximated distributions of the smallest magnitude case $\left(F_{y}\right.$ of load case 89$)$ and the highest magnitude case $\left(M_{y}\right.$ of load case 37$)$ are shown in Fig. 2 and Fig. 3 respectively. The approximations were constructed from 25 characteristic loads.

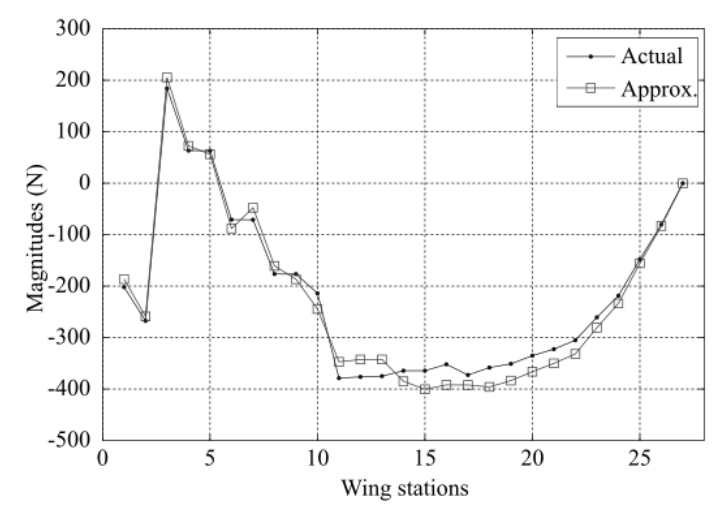

Fig. 2 Actual vs approximated $\boldsymbol{F}_{\boldsymbol{y}}$ (smallest magnitude component) (load case 89, $k=25$ )

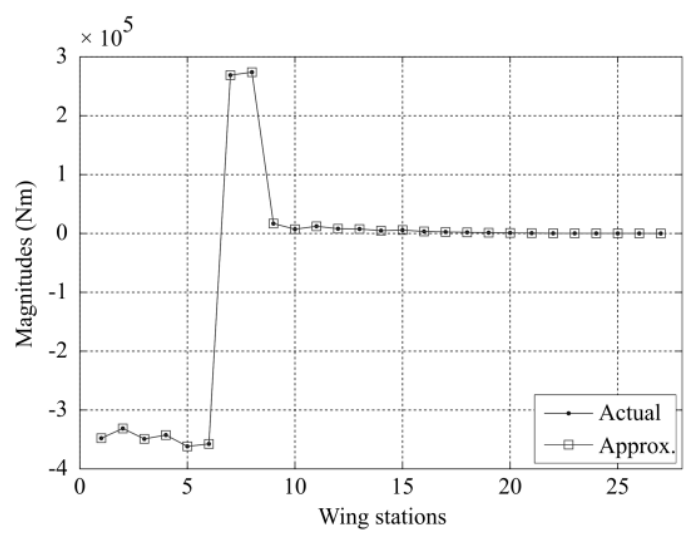

Fig. 3 Actual vs approximated $M_{y}$ (largest magnitude component) (load case 37, $k=25$ )

The error relative to the actual load value at each station may not be a good indicator as a small load value (which would be irrelevant when considering failure) will amplify the error significantly. Instead the Euclidean norm calculated from all load values at a particular station and degree of freedom (i.e. loads in the same column) of the load matrix, which represents the overall magnitude of every load case at that station, was used as a reference value. From Fig. 2 and Fig. 3, the errors at each wing station relative to the Euclidean norm of all the load values at each station are all below $2 \%$.

An important factor that should also be considered is when the total number of load cases grows larger. The experiment was conducted by including all the 274 load cases into the master set. The new size of the master set now becomes $A=(274 \times 162)$, so its effective rank has been altered. While the rank of the previous matrix is equal to the size of the rows $(r=100)$, the rank of the new matrix is equal to the size of the columns $(r=162)$. Therefore, the maximum number of characteristic loads $(k)$ has changed from 100 to 162 . 
The error when using 1 to 162 characteristic load cases to approximate 274 master load cases is shown in Fig. 4. There is no dramatic difference between the new error result compared to the previous one, where the number of the master load cases is 100 , for the first 20 characteristic loads. However, the difference grows further when the number of characteristic loads is increased. From Fig. 5, the SVD tends to preserve the overall trend of the singular values. However, adding more load cases in the $A$ matrix means that the diversity of the load case patterns will also be increased.

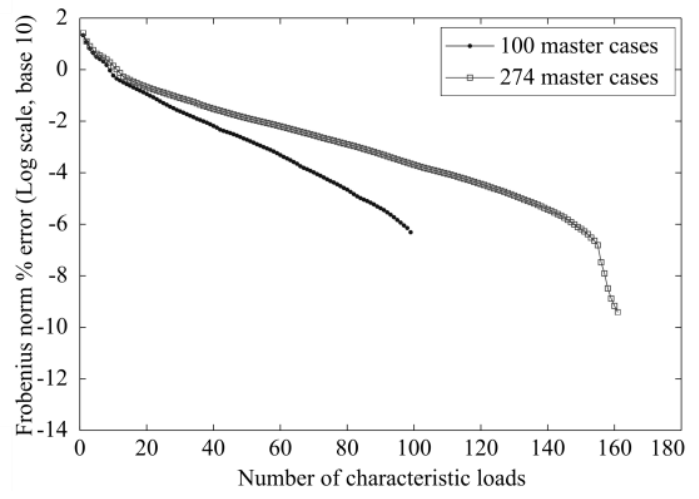

Fig. 4 Frobenius norm errors from estimating characteristic loads using all 100 load cases vs 274 master cases

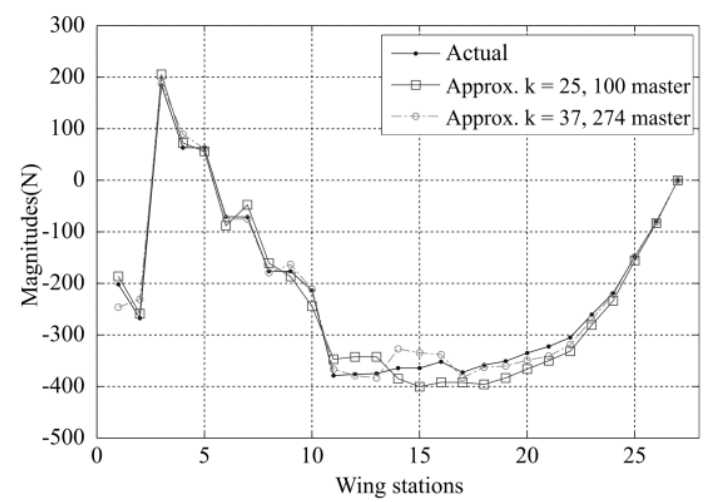

Fig. 5 Actual vs approximated $F_{y}$ (load case 89, $\boldsymbol{k}=$ 25 for 100 master cases vs $k=37$ for 274 master cases)

To obtain the accuracy at roughly the same scale as approximating 100 cases with $k=25$ characteristic loads, approximating 274 load cases requires $\approx 37$ characteristic loads. Fig. 5 displays $F_{y}$ distributions approximated from the SVD of 100 and 274 load cases using different $k$ values which produce roughly the same level of errors (same intersecting horizontal line in Fig. 4). The results show that the load distributions approximated from both methods are similar.

It can clearly be seen that the load case reduction ratio is lower in the 100 master cases than the 274 cases $(75 \%$ vs $86.5 \%)$. However, the rank reduction ratios $(k / r)$ of both load matrices are almost equal $(\approx 1 / 4)$. In either case, once the target accuracy is chosen $(0.1 \%$ in this case), the number of load cases required can be read from the intercept of the graphs in Fig. 4 with the appropriate horizontal line.

\section{H. Experiment on the wing external load cases - slave load cases}

A study with slave cases was conducted to represent the scenario when updated loading is added and there is a desire to represent these as linear combinations of the previously calculated characteristic loads of A. The general 
procedure is that characteristic loads $\left(L_{k}\right)$ are obtained from the SVD of $A$. Referring to Eq. (18), the pseudo inverse of the characteristic loads $\left(L_{k}^{\dagger}\right)$ calculated by a predefined function in MATLAB is then used to map the left eigenvector matrix $\left(\widetilde{U}_{k}\right)$ which can be used to estimate the new matrix $B_{k}$, Eq. 18.

The characteristic loads $L_{k}$ from the master cases that were used to estimate the load case matrix $B_{k}$ of the slave cases range from 1 to 100. The Frobenius norm error calculated from the difference between the approximate and the actual slave case matrices i.e. $\left\|B-B_{k}\right\|_{F}$ is shown in Fig. 6 . The results clearly show that the error does not become logarithmically small with increasing $k$ like the one in Fig. 4. Since $\widetilde{U}_{k}$ is not the actual $U$ extracted from the SVD, an error should always be expected. From the figure, the maximum accuracy that can be obtained is around $1 \%$.

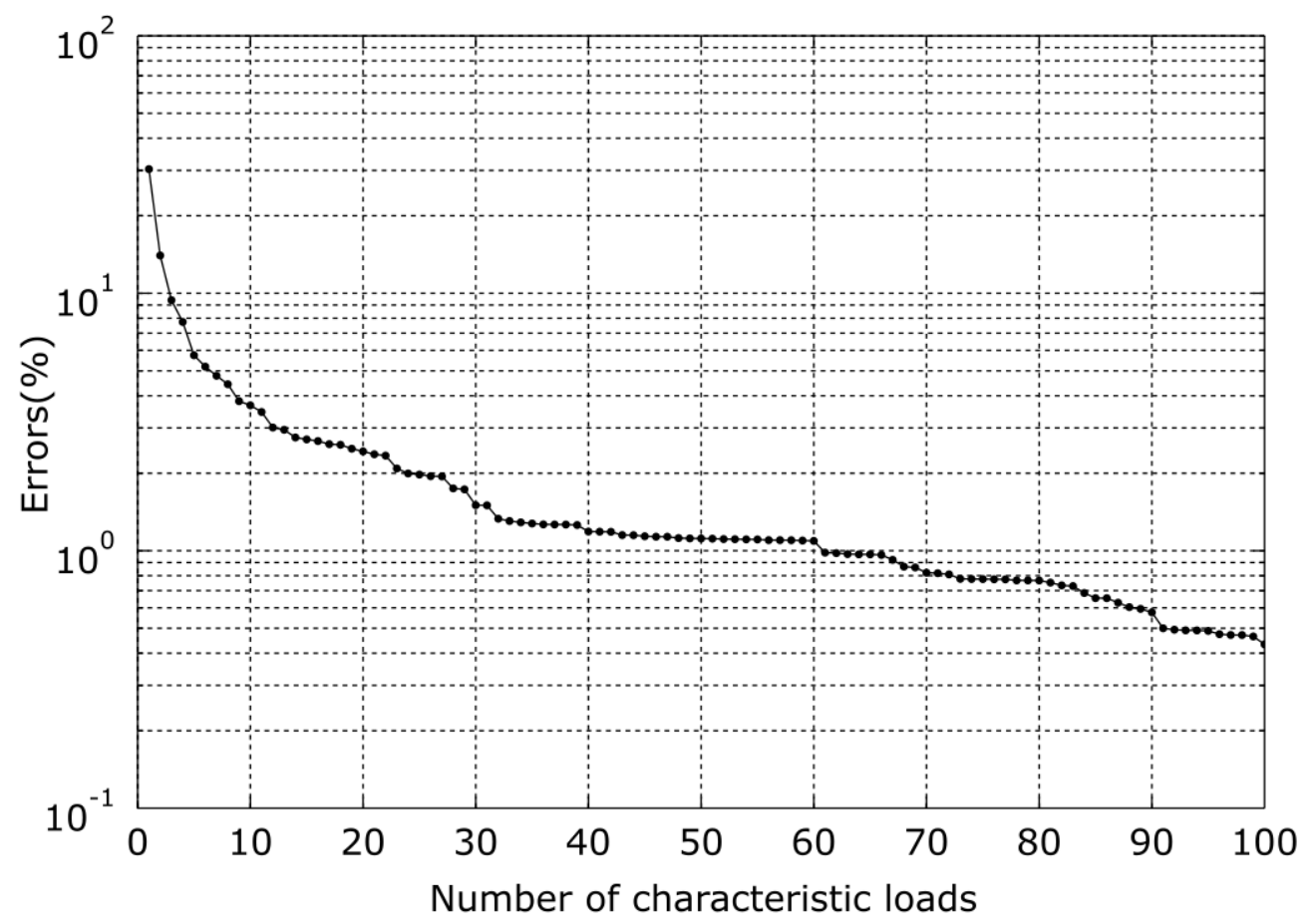

Fig. 6 Frobenius norm errors from estimating 174 slave cases using 100 characteristic loads

Unfortunately, because the method is equivalent to least square fitting of the linear equations, some of the solutions may not be accurate. This is because some load patterns, which are required to represent a particular slave case in $B$, are missing from the characteristic loads $\left(L_{k}\right)$ extracted from the master cases $(A)$. This behavior is not dependent on the magnitudes of each load case. Fig. 7 and Fig. 8 show the $F_{y}$ distributions of the slave load case 1 
and 121 using 100 characteristic loads where the maximum accuracy of the reduced rank approximation should be expected. The magnitude of the slave case 1 is around 10 times higher than the load case 121 but was very poorly reconstructed.
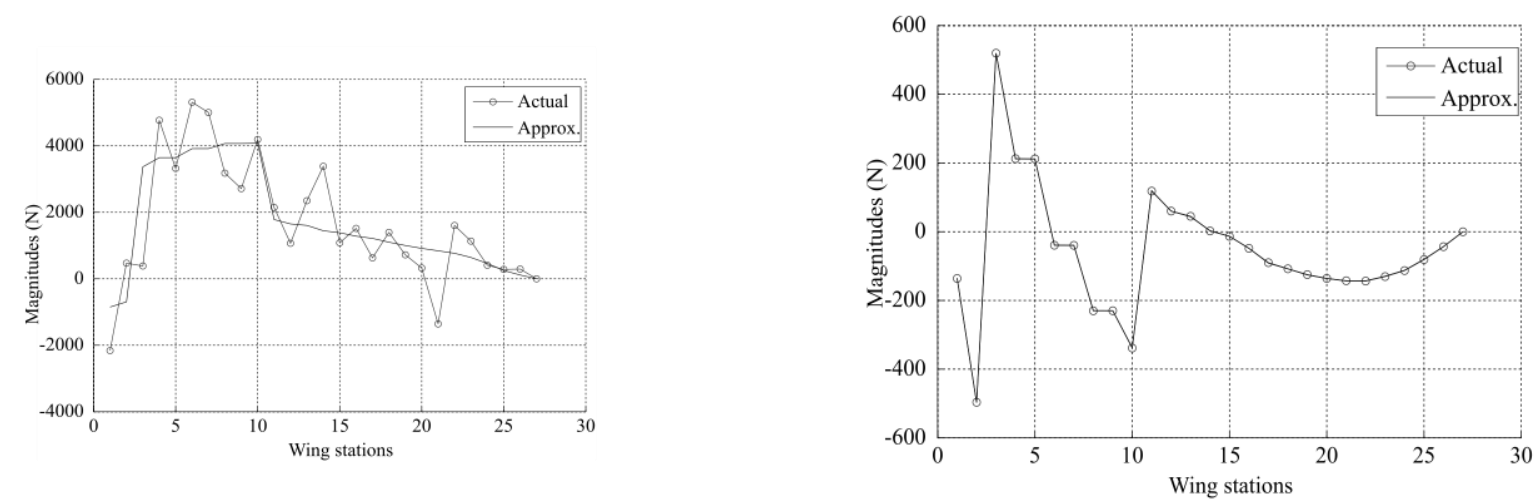

Fig. 7 Actual vs approximated $F_{y}$ (slave load case 1, Fig. 8 Actual vs approximated $F_{y}$ (slave load case $\boldsymbol{k}=\mathbf{1 0 0}$ ) $121, k=100$ )

One of the effective ways to filter out the load cases that are poorly estimated is to use all the characteristic load cases i.e. $k=100$ to evaluate the error and create a histogram plot. Then the variation among the errors should implicitly indicate the region where most of the data, which is accurately fitted, lies. The histogram displaying the normalized error of each load case is shown in Fig. 9. 


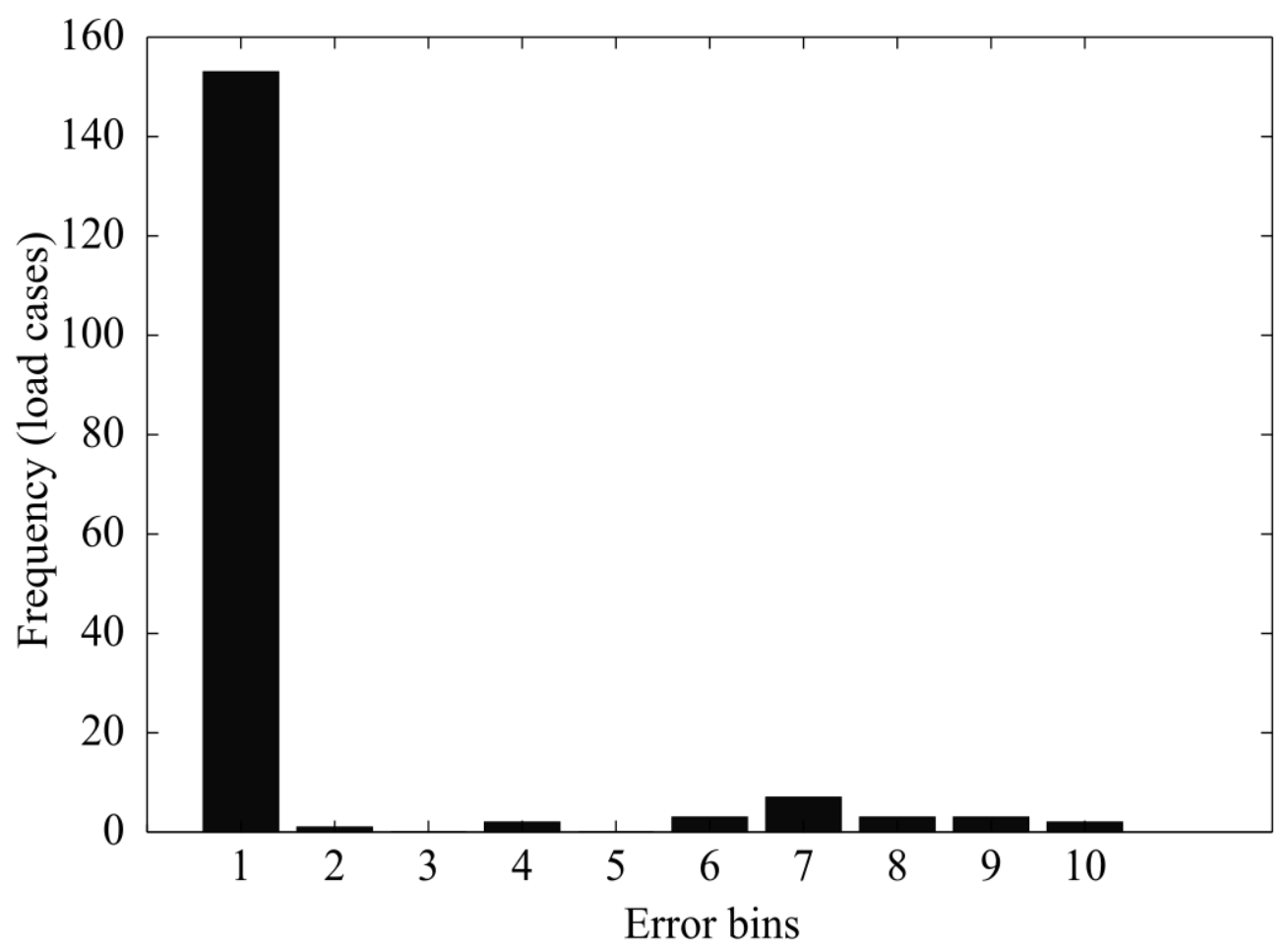

Fig. 9 Histogram of relative true error per station of all slave cases, each bin represents a $\Delta 3 \%$ error ( $k=$ 100, master $=100$ )

From Fig. 9, $154(\approx 88 \%)$ of the total 174 slave cases in the first bin have an average normalized error below 3\% per load case. If the error threshold is set using the first bin i.e. 3\%, 20 load cases are not satisfactorily approximated. The omitted load cases can be treated separately provided that the first set of characteristic loads has been fixed, or they can be directly included into the master set as already demonstrated, so that computing a new SVD on all available load cases required only ten extra load cases. This therefore provides a procedure which indicates a) if the new slave cases are satisfactorily approximated by the existing SVD and b) which slave cases need to be added to the master set if the approximation is not satisfactory.

\section{Analyzing Characteristic Load Cases}

In the aircraft structural design process a large number of external load cases must be analyzed in the Global Finite Element Model (GFEM). Each external load case is evaluated in the GFEM to attain internal load paths going into each sub-structure. Since it is not possible to analyze all generated load cases, a down-selection process is performed before the load cases are analyzed in GFEM. However, the lack of a robust down-selection process 
makes the current design process inefficient and too dependent on engineering judgement. From the last section, it is shown that the SVD is able to identify a few characteristic load cases that represent all external loads. This section will show that the analysis results from these characteristic load cases can be linearly combined with using the SVD equations above to create the internal loads resulting from all external load cases, hence eliminating the downselection process.

\section{A. Global Finite Element Model (GFEM)}

A GFEM is generally a low degree of fidelity finite element model representing an aircraft structure. The majority of a GFEM is a collection of idealized frames, stringers and panels which are represented by relatively coarse elements i.e. shell and bar elements. An example of an industry standard GFEM used for this study is shown in Fig. 10.

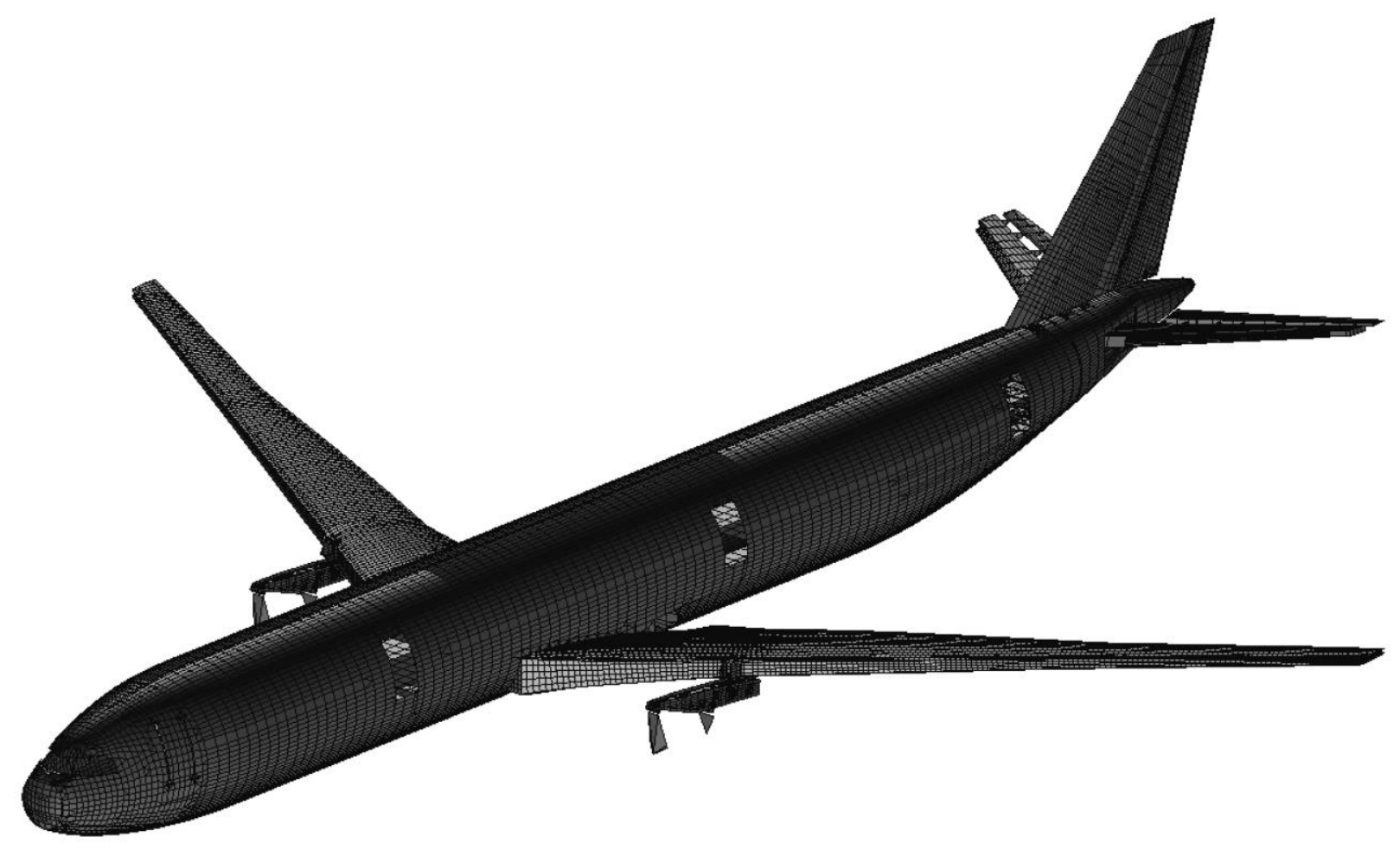

Fig. 10 Industrial standard aircraft GFEM

Each major component e.g. wing, fuselage and horizontal tail plane is often analyzed separately with some boundary conditions defining the structural constraints. In this paper external load cases were analyzed on the right wing structure separated from the full GFEM. The external load cases can be applied directly to this model for each 
reference station on the wing. The reference stations have Multi-Point Constraint (MPC) equations which link the degrees of freedom of the reference station grid points to the grid points of each rib.

Generally once all the external loads have been analyzed in the GFEM, the load paths or the internal loads can subsequently be requested as an FEA output of each structural element, which is an idealized stiffened skin panel segment sometimes called a super-stiffener. This type of structure is of importance for the sizing of the wing as it accounts for more than $50 \%$ of its structural weight.

\section{B. Internal load analysis in GFEM}

Generally in finite element analysis, a linear response such as an internal load $(N)$ due to an applied external force matrix $(F)$ on a GFEM is linearly related by a single matrix $(M)$ as

$$
N^{T} \approx M F
$$

such that $M$ matrix involves global and local structural stiffness matrices of the structure.

In order to reduce the number of load cases required to be analyzed using SVD, the $F$ matrix must be rearranged to the same format as the $A$ matrix. This can be done by multiplying with a permutation matrix $(P)$, which is a square matrix where each row and column contains a single "1" and "0" elsewhere [12], so that the resulting GFEM loads $F$ are $P A^{T}$. Equation (19) then becomes

$$
N^{T}=M P A^{T}
$$

This equation indicates that an internal load matrix $N$ has a linear relationship with the load case matrix $A$. The $N$ result on any structural feature can be obtained directly by applying $A$ on a GFEM.

\section{Superposition of characteristic loads}

Since the relationship between the internal loads and the external load matrix has already been established, the characteristic loads extracted from SVD can be integrated into the GFEM analysis. As previously shown, the $A$ matrix can be decomposed by the SVD into characteristic loads $L^{T}$ and the corresponding $U^{T}$ matrices, Eq. (20) can be replaced by

$$
N^{T}=M P L^{T} U^{T}
$$

Applying the reduced rank approximation yields

$$
N^{T} \approx M P L_{k}^{T} U_{k}^{T}
$$


Eq. (22) represents the superposition of characteristic loads, which is applicable for any finite element applications whose requested outputs are linearly related to the model. This equation can be described as rearranging $L_{k}^{T}$ into the new format specified by the permutation matrix $(P)$ and evaluating the load cases the matrix in finite element analysis. The result is the internal loads matrix, $M P L_{k}^{T}$. The linear combination between $M P L_{k}^{T}$ and $U_{k}^{T}$ should produce the similar result as analysing $A^{T}$ in Eq. (20), provided that the reduced rank is sufficient.

Eq. (22) shows that the internal loads $\left(N^{T}\right)$ for the full set of load cases in $A$ can be obtained by linearly combining the $k$ analysis results from characteristic loads $\left(L_{k}\right)$ and the corresponding left eigenvector matrix $\left(U_{k}\right)$.

\section{Analyzing external wing characteristic load cases - description}

The model used is a representative wing GFEM detached from a full aircraft GFEM. The original and the external characteristic 6DOF force and moment load cases were decomposed and analysed in linear finite element analysis, which were carried out in MD Nastran 2011 (SOL101) to obtain the internal loads.

The resulting internal loads were checked on three different structural elements located near the wing root, middle and tip on the top skin as shown in Fig. 11. Each structural element consists of two idealised panels consisting of four shell elements each and one stringer consisting of four bar elements. The $x$-axis is in the span-wise direction, which aligns with the stringer. All shell elements are governed by three in-plane forces $\left(N_{x}, N_{y}, N_{x y}\right)$, and the bar elements are only governed by an axial force $\left(P_{x}\right)$. The forces will be averaged in each panel and stringer. In total there are seven force components i.e. three in-plane forces on both panels and one axial force on a stringer.

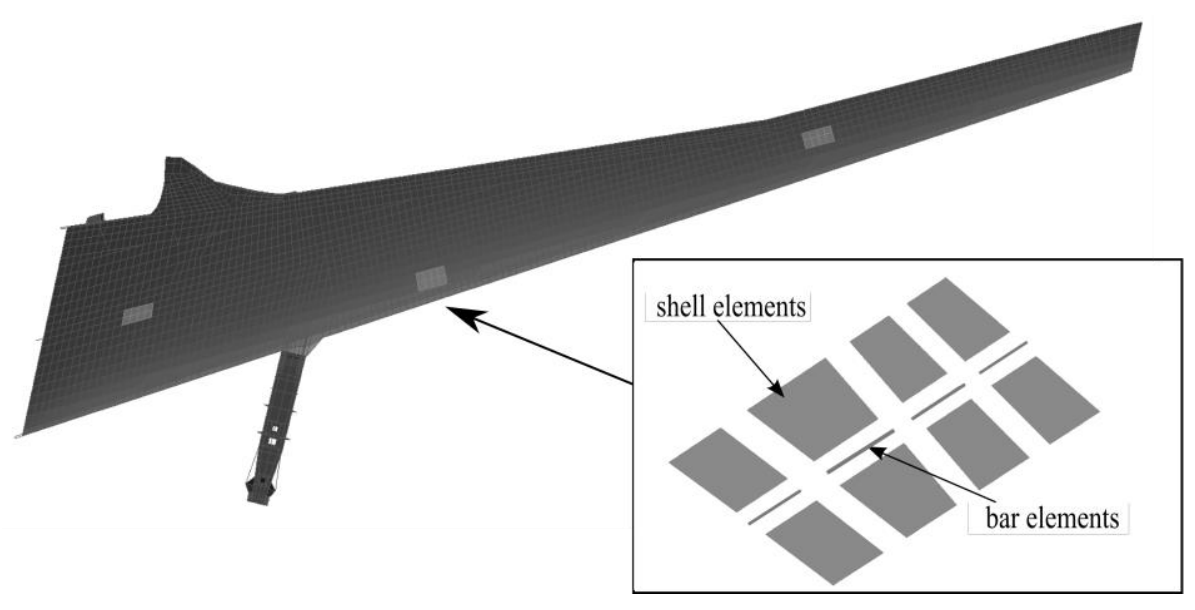

Fig. 11 Three idealized structures, super-stiffeners, on a wing GFEM 
The 100 wing characteristic load cases $\left(L_{k}\right)$ from the previous section were run in Nastran. Their analysis results were linearly combined with the corresponding left eigenvector matrix $\left(U_{k}\right)$ to create 100 analysis results. The linear combination can easily be achieved in matrix manipulation programs such as MATLAB or Python (NumPy) with almost negligible computational time. The comparison of the reconstructed internal loads with the actual internal loads will be shown along with its reserve factor values.

Finally, all characteristic loads $\left(L_{k}\right)$ extracted from the master set were linearly combined with $U_{k}$ estimated from the least squares method to create the analysis results of all 174 slave cases. The slave set is used to demonstrate the ability of the least squares method to estimate the loads that are not the product of the SVD. The experiment was conducted in the same manner as the master set.

\section{E. Analyzing external wing characteristic load case - master case}

The internal load results for different numbers of characteristic loads ( $k=1$ to 100$)$ were extracted at the three chosen structural elements. The analysis results were then linearly combined with the corresponding $U_{k}$ matrices to approximate the internal loads of each individual load case $\left(N_{k}\right)$. Similarly, the actual internal loads $(N)$ were obtained by running the full original 100 load cases $(A)$.

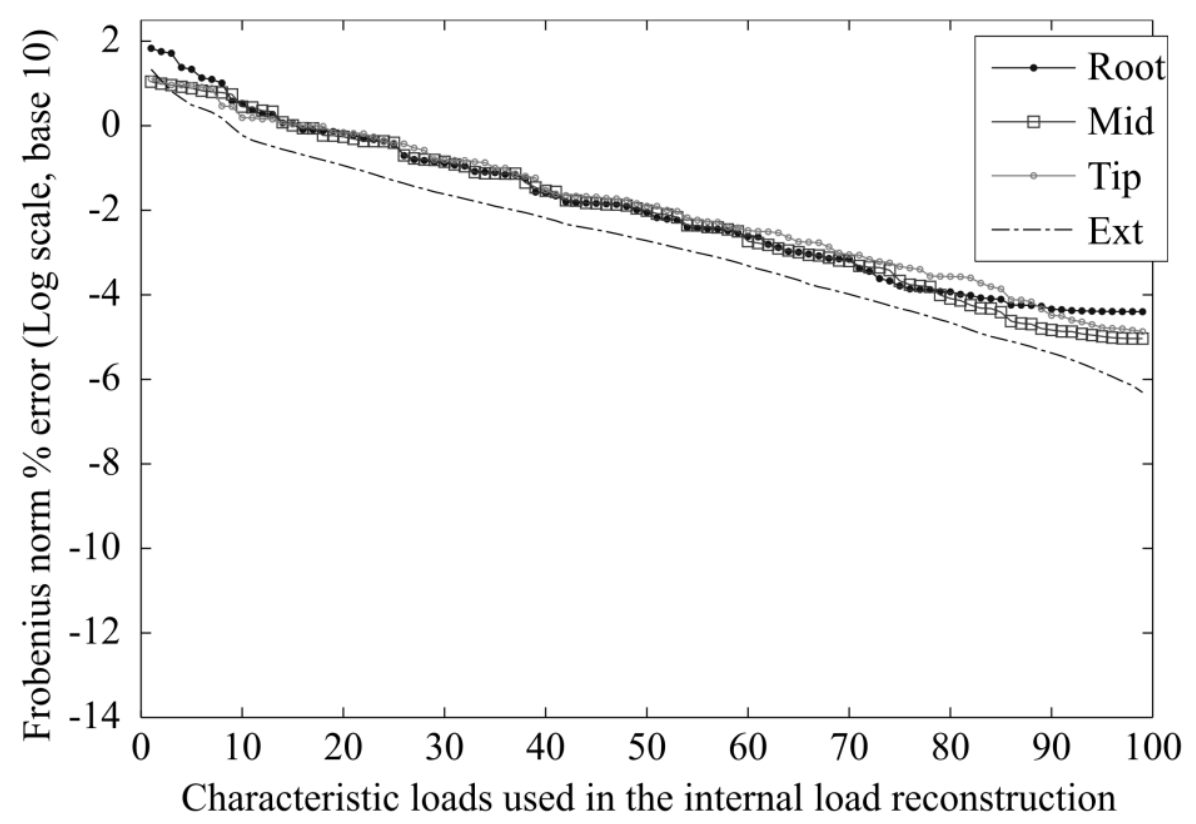

Fig. 12 Frobenius norm \% errors of external loads (Ext) and the corresponding internal loads on superstiffeners at wing root (Root), midpoint (Mid) and tip (Tip), $k=1, \ldots, 99$ (log scale) 
The Frobenius norm percentage error in the log scale with base 10 is shown in Fig. 12. The errors from the superposition of characteristic loads possess a similar trend to the approximation of the external loads. The errors for all three structural elements are almost in the same line. The external load error appears to be their minimum bound.

The internal load distribution in each individual structural element was also investigated. As one might expect, the extreme external load cases cause the extreme internal loads in the local structures. Fig. 13 represents the individual internal load components of the smallest external load case (89) on the structural element near the wing root. The $x$ axis consists of 7 load components, and has been arranged in the format of:

$$
\left(N_{x}, N_{y}, N_{x y}\right)_{\text {right panel }}\left(N_{x}, N_{y}, N_{x y}\right)_{\text {left panel }}\left(P_{x}\right)_{\text {stringer }}
$$

where each load is averaged from all forces in a set of four finite elements. All the panel's forces $\left(N_{x}, N_{y}, N_{x y}\right)$ have different units from the stringer's axial force $\left(P_{x}\right)$ i.e. Newton per unit length, so they have been scaled up for visualization purposes.

In Fig. 13, the internal load results reconstructed from $k=25$ are very accurate while $k=35$ gives almost exactly the same results as the original internal loads. Using $k=10$ produces a poorly reconstructed $N_{x}$ on both panels and $P_{x}$ on the stringer. Fig. 14 represents the internal loads due to the highest bending case (37) on the structural element near the wing root. All internal load results are accurately reconstructed as expected, even with just 10 characteristic loads or $10 \%$ of the original data.

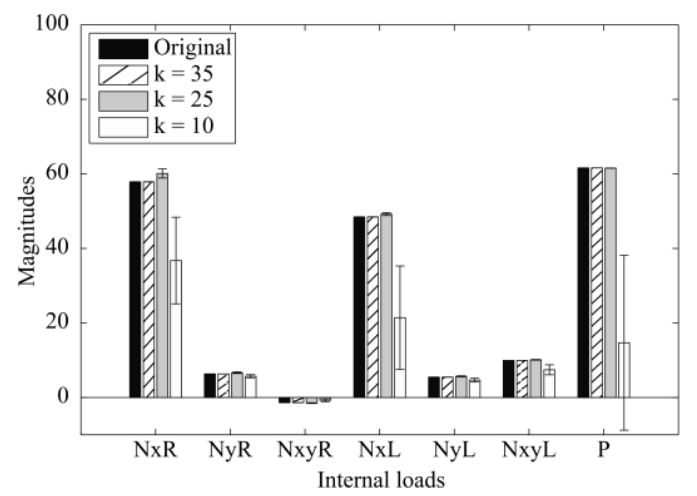

Fig. 13 Internal loads (Tip SE, load case 89)

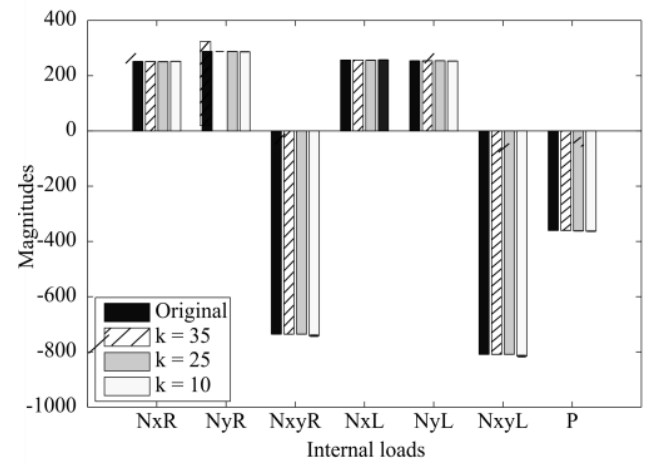

Fig. 14 Internal loads (Root SE, load case 37)

Therefore, the experiment clearly indicates that 25 characteristic loads are adequate to accurately reconstruct all internal load resulting from the 100 master load cases from which they have been extracted. After normalizing the error at each station by the Euclidean norm of all internal loads at that station, the reconstruction errors of every internal load value are well below $1 \%$ for all 3 structural elements. Even in the extreme scenario i.e. using $10 \%$ of 
the original load cases, the approximation could predict the critical cases reasonably well. The small inaccuracy of the external load should also barely impact structural failure calculations, as will be further discussed below.

\section{F. Analyzing external wing characteristic load case - slave case}

The superposition technique is also applicable for the new load cases. $\widetilde{U}_{k}$ which is an eigenvector matrix estimated from the least squares approach using Eq. (18) is already available. The internal loads of all 174 slave cases can be superimposed from the analysis results from the k characteristic loads. The Frobenius norm percentage resulted by superimposing the slave cases is shown in Fig. 15.

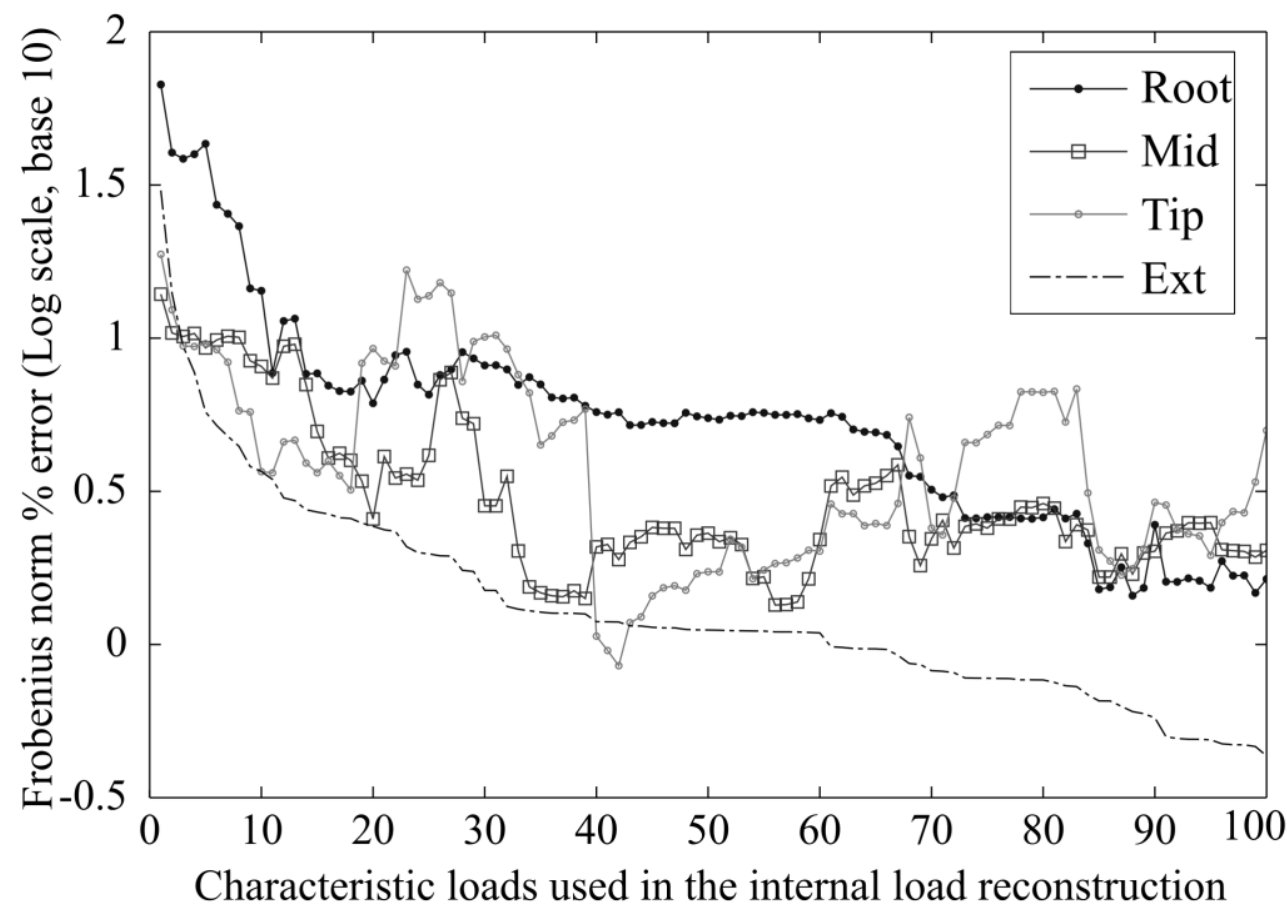

Fig. 15 Frobenius norm \% errors, $k=1, \ldots, 100$ (log scale)

The slave external load error is the minimum bound of the internal load errors. However, the internal load errors fluctuate throughout the entire data range, especially for the structural elements near the middle and tip of the wing. The error at the wing root structural element located is generally the highest - due to its sizing it has low internal load magnitudes compared to the others.

As previously explained, there might be some new external load cases that cannot be accurately estimated by the least squares method and should either be included into the original master set or treated separately. The comparison 
of the actual internal loads and the internal loads superimposed using three different sizes of characteristic loads $(k=$ 25, 35, 100) for slave load cases 1 and 121 are shown in Fig. 16 and Fig. 17 respectively.

From Fig. 7, slave case 1 was poorly estimated by the least squares method despite its relatively high magnitudes. The error of its internal loads shown in Fig. 16 is noticeably high despite its very high internal load magnitudes. Even when using all the characteristic loads, the error is still relatively high. Thus, this load case should definitely be excluded from the least squares method as already suggested. The GFEM needs to be run again for this load case. Considering Fig. 17, the internal loads of the slave case 121 were accurately estimated when using just 25 characteristic loads despite its relatively low magnitude, as expected by its accurate external load approximation (Fig. 8).

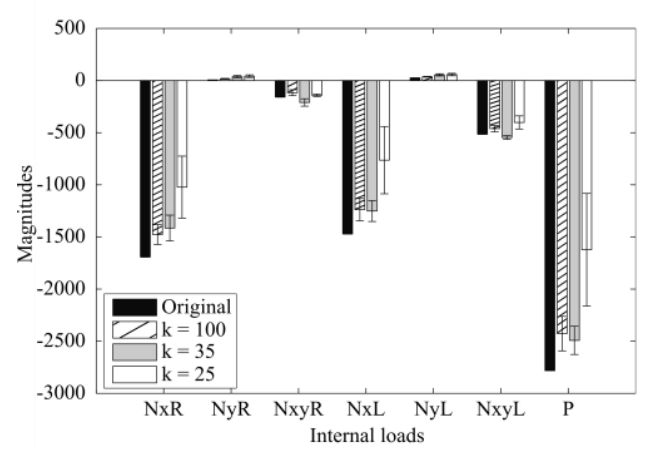

Fig. 16 Internal loads (Tip SE, slave case 1)

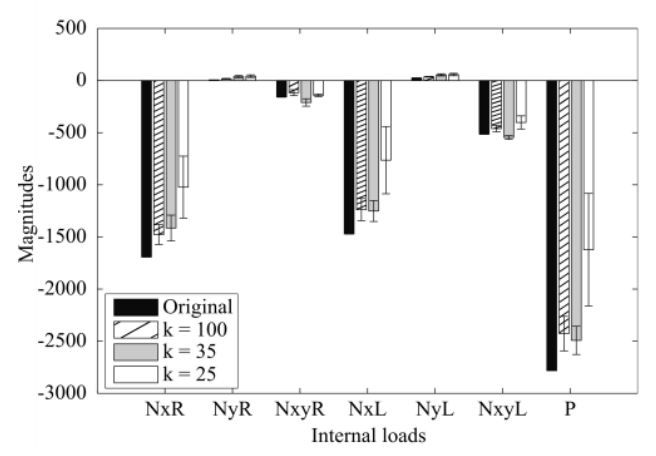

Fig. 17 Internal loads (Root SE, slave case 121)

\section{G. Reserve factor comparison}

The goal of a GFEM analysis is to provide the internal load distributions that allow a local stress evaluation of whether each local structure can withstand the applied load cases, in terms of reserve factors (RF). To implement the characteristic loads onto the GFEM analysis, it is important to ensure that the inaccuracy in the reconstructed internal loads will not excessively affect the RFs. The comparison of RFs resulted from evaluating the actual internal loads $(N)$ and the re-constructed internal loads $\left(N_{k}\right)$ under the given failure criteria, which were provided by Airbus (UK) as an executable function. Such criteria include local buckling, global buckling and damage tolerance.

Based on the previous experiment, 25 characteristic loads were selected since they can reconstruct the internal loads accurately. Since the large RFs are of least interest and the smallest RFs are of most interest, the graphs compare 1/RF values. The comparison of $1 / \mathrm{RF}$ computed using actual and approximated load cases for the middle and tip structural elements are shown in Fig. 18 and Fig. 19. The points with high RFs will be in the lower region of 
the plot, and the critical RFs are the ones close to 1 . The straight line indicates the value when the approximated RF is exactly equal to the actual RF. All RFs are close to the exact line, meaning that $k=25$ characteristic loads captured all the load cases and their effect on RF.

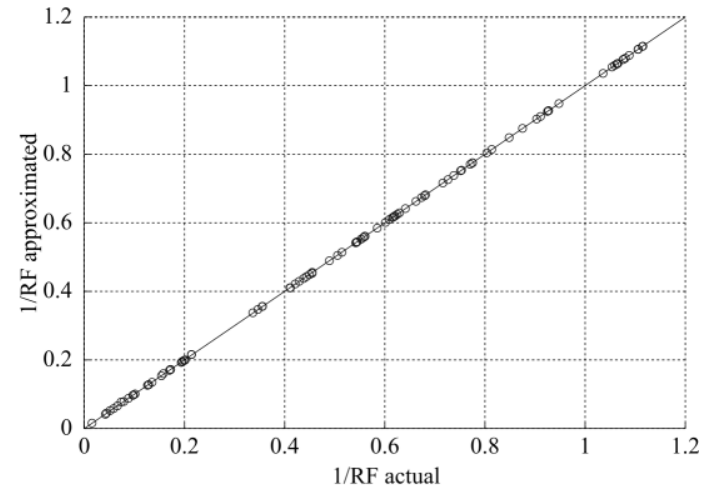

Fig. 18 1/RF comparison (Mid SE, 100 master cases)

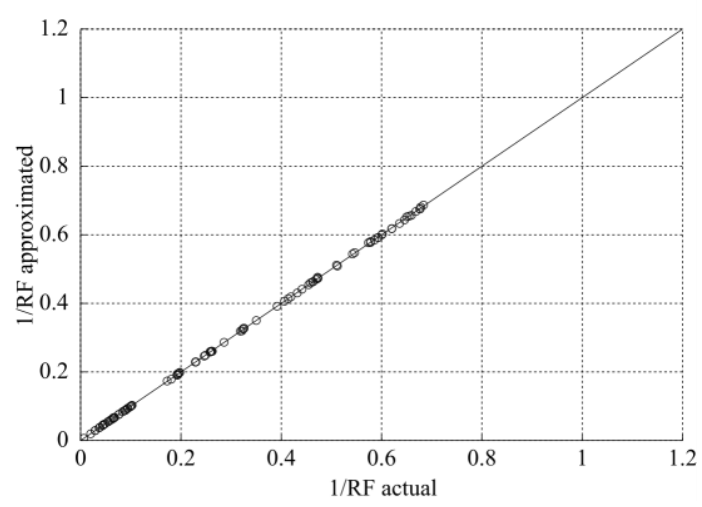

Fig. 19 1/RF comparison (Tip SE, 100 master cases)

The accuracy of the slave load cases was also studied on the wing root structural element. The reserve factors of the 154 slave cases which were reconstructed accurately by the least squares method are plotted in Fig. 20. The other 20 cases, which should be omitted from the least squares method, are plotted on Fig. 21. The plots clearly indicate that the least squares estimations of all the 154 slave cases, whose approximation errors at the external load level are below the threshold, are accurate. All the low RF values are clustered close to the exact line.

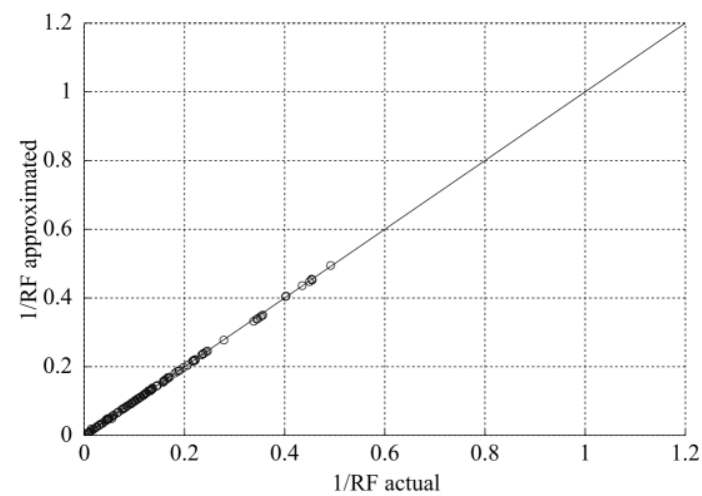

Fig. 20 1/RF comparison (Root SE, good cases)

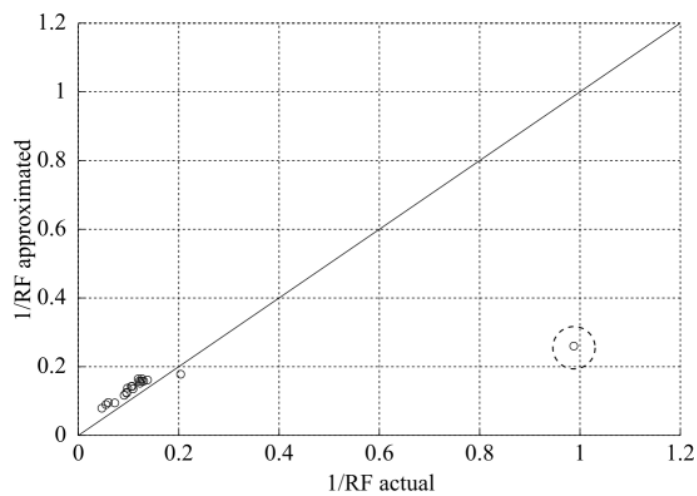

Fig. 21 1/RF comparison (Root SE, poor cases)

Considering the plots of the omitted slave cases, almost all load cases that are poorly reconstructed have very high RF values. These load cases may not affect the structural failure at all. Therefore, there might be only one load case (slave case 1) where an additional GFEM load case would be necessary. 


\section{Discussion}

In this paper, a characteristic load approach based on the SVD of the load case matrix has been proposed. The SVD reduced rank approximation allows a small set of characteristic loads which can represent the full range of load cases to be derived. It was found that a number of characteristic loads, equaling to about a quarter of the rank of the load case matrix, can be linearly combined to approximate all the original load cases. The approach can also be extended to the situation where new load cases may be provided during the design process, and the appropriate combination of existing load cases can be identified. Some exceptional cases may be experienced where the reconstruction is not accurate, but such load cases can be identified by computing the reconstruction error, which indicates when a new GFEM load case should be run. The Frobenius norm error is an efficient way to estimate the approximation of the entire load case matrix due to the reduced rank approximation. Small external load errors have a negligible influence on the computation of critical reserve factors using an industry-standard 'black box' RF evaluation.

The characteristic loads obtained from the external loads are very beneficial in the preliminary sizing stage. The GFEM load path, which is analyzed in each sizing loop, can be computed with minimal effort using the superposition of the internal loads resulting from the characteristic loads and the SVD's left eigenvalue matrix, which may be interpreted as the load coefficient. The error in external load provides a lower bound on the error in internal load. This is of course less useful than an upper bound, but the comparison plots between the Frobenius norm errors of the external and internal loads show that the actual and lower bound error appear to be closely related.

The analysis results show that the approximation error barely affects the critical load cases, as demonstrated by the high precision of the approximation of low reserve factor values. The trend is similar for new load cases estimated from the least squares method.

Alternative approaches are certainly possible. In [13] a neural network approach was used to select critical load envelopes. The surrogate model was trained using 2000 analyses based on variations in material and structural properties, load fluxes, control surface deflections and RF values to provide a cheap-to-evaluate approximate RF evaluation, which in turn allowed the efficient estimation of critical external load (shear, moment and torque) envelopes. A similar approach was used in [14] to assess the sensitivity of buckling RFs to maneuver load alleviation parameters. In addition, Tartaruga et al. [15] used SVD methods to explore the sensitivity of load 
envelopes to variations in design parameters such as engine mass and pylon stiffness during gust loading. The main philosophical differences of the present approach are a) the load quantities of interest emerge naturally from the SVD analysis rather than being chosen as $1 \mathrm{D}$ or $2 \mathrm{D}$ envelopes of 'interesting quantities' and $\mathrm{b}$ ) there is a direct, rather than surrogate, link between the loads and RF analyses. The relative cost, complexity and generality of the different approaches has still to be clarified.

The amount of computational reduction of the external load cases which need to be analyzed in the GFEM is equal to the SVD rank reduction ratio. Whilst the savings due to reduction in the number of GFEM load cases may seem relatively unimportant in the modern era of HPC, the reduction in the dimensionality of the load space becomes much more significant in other areas such as the calculation of design sensitivities, or the generation of failure envelopes for structural features.

\section{Future work}

In a future paper the approach developed here will be utilized to develop a failure envelope for structural features such as super-stiffeners in a 'characteristic load space' of low dimensionality. Using this, the non-redundant failure modes limiting performance can be identified and all the redundant failure constraints can be eliminated. This has very significant benefits in terms of both structural understanding and fast evaluation of RF values under a large number of load cases. The method also has application in gradient optimization, where a significant computational reduction in the computation of sensitivities of loads with respect to design variables can be achieved.

The immediate work is to correlate the physical type (ground, maneuver, gust etc.) of each global load case to the characteristic loads. This might assist in identifying the master set containing the minimum number of cases that is sufficient for spanning the load space.

The robustness of the method should also be improved. It would be interesting to see how the SVD can be applied to a much larger number of load cases. More stressing criteria should be tested, especially against some of the load cases which might initiate different type of failures in the structure. The effect when the types of structural elements and boundary conditions are changed must also be investigated.

Finally, it would be interesting to investigate how these ideas could be extended to non-linear analyses. Whilst the reduced rank approximations appear satisfactory for evaluating internal load and RF values, the neglected small variations in load distribution might have a significant effect on phenomena such as imperfection sensitivity or post- 
buckling analysis. Reduced order modelling is of great current interest in other areas such as CFD, so there will doubtless be opportunities to exploit these developments.

\section{Acknowledgements}

Financial support from Airbus (UK) Ltd for the PhD research of A Dharmasaroja is gratefully acknowledged.

\section{References}

[1] T. L. Lomax, Structural Loads Analysis for Commercial Transport Aircraftt: Theory and Practice, AIAA, 1996.

[2] J. R. Wright and J. E. Cooper, Introduction to Aircraft Aeroelasticity and Loads, Chichester: John Wiley \& Sons Ltd, 2007.

[3] H. H. Khodaparast, G. Georgiou, J. Cooper, L. Riccobene, S. Ricci, G. Vio and P. Denner, "Efficient Worst Case "1-Cosine" Gust Loads Prediction," Aeroelasticity and Structural Dynamics, vol. 2, no. 3, 2012.

[4] W. A. Stauffer, J. G. Lewolt and F. M. Hoblit, "Application of Advanced Methods to Design Loads Determination for the L-1011 Transport," Journal of Aircraft, vol. 10, no. 8, pp. 449-458, 1973.

[5] K. Appa, "Recent advances in maneuver loads analysis," Computer Methods in Applied Mechanics and Engineering, vol. 90, no. 1, pp. 693-717, 1991.

[6] E. Ünay, D. Gürak, V. Özerciyes, A. Uzunoğlu and H. Kestek, "Tool developement for aircraft loads postprocessing," in 28th Congress of the International Council of the Aeronautical Sciences, Brisbane, 2012.

[7] S. McGuinness, PhD thesis: Improving Aircraft Stress-Loads Interface and Evaluation Procedures, Queens University Belfast, 2011.

[8] G. W. Stewart, "On the early history of the singular value decomposition," SIAM Rev., vol. 35, no. 4, pp. 551$566,1993$.

[9] C. Eckart and G. Young, "The approximation of one matrix by another of lower rank," Psychometrika, vol. 1, no. 3, pp. 211-218, 1936.

[10] G. Golub and C. Van Loan, Matrix Computations, 3rd ed., Johns Hopkins University Press, 1996.

[11] A. Ben-Israel and T. N. Greville, Generalized Inverses: Theory and Applications, 2 ed., Newyork: Springer- 
Verlag, 2003.

[12] R. Brualdi, Combinatorial Matrix Classes, Cambridge University Press, 2006.

[13] R. Nazzeri, M. Haupt, F. Lange and C. Sebastien, "Selection of critical load cases using an artificial neural network approach for reserve factor estimation," in Deutscher Luft- und Raumfahrtkongress, Rostock, 2015.

[14] R. Nazzeri, F. Lange and M. S. C. Haupt, "Assessing sensitivities of maneuver load alleviation parameters on buckling reserve factors using surrogate model based extended Fourier amplitude sensitivity test," in 11th World Congress on Structural and Multidisciplinary Optimisation, Sydney, Australia, 2015.

[15] I. Tartaruga, J. Cooper, M. Lowenberg, P. Sartor, C. S and Y. Lemmens, "Prediction and uncertainty propagation of correlated time-varying quantities using surrogate models," CEAS Aeronautical Journal, vol. 7, no. 1, pp. 29-42, 2015. 